\title{
SPECTRUM OF THE PRODUCTS OF OPERATORS AND COMPACT PERTURBATIONS
}

\author{
WEIBANG GONG AND DEGUANG HAN
}

(Communicated by Palle E. T. Jorgensen)

\begin{abstract}
In this paper we will give a complete characterization of the operator $B$ which satisfies the spectral condition $\sigma(A B)=\sigma(B A)$ (resp. $\sigma_{e}(A B)=$ $\left.\sigma_{e}(B A)\right)$ for every $A$ in $L(H)$ and also a spectral characterizations of the product of finitely many normal (resp. essentially normal) operators.
\end{abstract}

\section{INTRODUCTION}

Let $H$ be an infinite-dimensional complex Hilbert space, $L(H)$ be the algebra of all bounded linear operators on $H$, and $K(H)$ be the compact operator ideal of $L(H)$. For $B$ in $L(H), \sigma(B)$ and $\sigma_{e}(B)$ denote the spectrum and the essential spectrum of $B$ respectively.

For $B$ in $L(H)$, we say that $B$ is consistent in invertibility (with respect to multiplication) or, briefly, a CI operator if, for each $A$ in $L(H), A B$ and $B A$ are invertible or noninvertible together. By Jacobson's Theorem (for $A, B \in$ $L(H)$, the nonzero elements of $\sigma(A B)$ and $\sigma(B A)$ are the same), $B$ is a CI operator if and only if $\sigma(A B)=\sigma(B A)$ for every $A$ in $L(H)$. Thus if $A$ and $B$ are CI operators, then so is $A B$. Our problem is: which elements in $L(H)$ are CI operators? Paper [6] caused the authors to think that this is a significant problem and remains to be considered.

The main purpose of this paper is to give a complete characterization of $B$ in $L(H)$ which satisfies the spectral condition $\sigma(A B)=\sigma(B A)$ (resp. $\sigma_{e}(A B)=$ $\left.\sigma_{e}(B A)\right)$ for every $A$ in $L(H)$. We also give a complete characterization of the $\mathrm{CI}$ operators which are invariant under compact perturbations.

\section{FUNDAMENTAL THEOREM}

Every $B$ in $L(H)$ must be included in one of the following five cases, and in each of them the problem is definitely answered. Theorem 11.2 in [7, p. 224] is useful in the proofs below.

Case 1. If $B$ is invertible, then $B$ is a $\mathrm{CI}$ operator.

Proof. It is sufficient to note that for every $A$ in $L(H), A B=B^{-1}(B A) B$.

Received by the editors December 10,1991 and, in revised form, June 11, 1992.

1991 Mathematics Subject Classification. Primary 47A10, 47A05, 47A55.

Key words and phrases. Spectrum, compact perturbation, normal operator, essentially normal operator, BDF theorem. 
Case 2. If $\operatorname{ran} B$ is not closed, then $B$ is a $\mathrm{CI}$ operator.

Proof. It follows from $\operatorname{ran} B A \subseteq \operatorname{ran} B \subset \overline{\operatorname{ran} B} \subseteq H$ for every $A$ in $L(H)$ that $B A$ is not invertible.

It is now to be proved that, for every $A$ in $L(H), A B$ is also not invertible. If, for some $A \in L(H), A B$ were invertible, the expression $(A B)^{-1} A B=$ $(A B)^{-1}(A B)=I$ indicates that $B$ is bounded from below. Then $\operatorname{ran} B$ is closed, which contradicts the assumption.

Case 3. If $\operatorname{ker} B \neq 0$ and $\overline{\operatorname{ran} B} \subset H$, then $B$ is a CI operator.

Proof. For each $A$ in $L(H), \operatorname{ker} A B \supseteq \operatorname{ker} B \neq 0$ implies that $A B$ is not invertible and $\operatorname{ran} B A \subseteq \overline{\operatorname{ran} B} \subset H$ implies that $B A$ is not invertible.

Case 4. If $\operatorname{ker} B=0$ and $\operatorname{ran} B=\overline{\operatorname{ran} B} \subset H$, then $B^{*} B$ is invertible while $B B^{*}$ is not invertible, and so $B$ is not a CI operator.

Proof. It follows from $\operatorname{ran} B B^{*} \subseteq \operatorname{ran} B \subset H$ that $B B^{*}$ is not invertible.

Since $B$ has closed range if and only if $B^{*} B$ has (see [2]), this together with the fact that $B^{*} B$ is one-to-one and has dense range implies that $B^{*} B$ is invertible.

Case 5. If $\operatorname{ker} B \neq 0$ and $\operatorname{ran} B=\overline{\operatorname{ran} B}=H$, then $B^{*} B$ is not invertible while $B B^{*}$ is invertible, and so $B$ is not a $\mathrm{CI}$ operator.

Proof. It follows from $\operatorname{ker} B \neq 0$ and $\operatorname{ran} B=\overline{\operatorname{ran} B}=H$ that $\operatorname{ker} B^{*}=0$ and $\operatorname{ran} B^{*}=\overline{\operatorname{ran} B}^{*} \subset H$. Therefore, by replacing $B$ by $B^{*}$ in the proof of Case 4, we obtain that $B^{*} B$ is not invertible and $B B^{*}$ is invertible.

By the results just proved above we can conclude

Theorem 1.1. $B \in L(H)$ is a CI operator if and only if one of the following three mutually disjoint cases occurs:

(1) $B$ is invertible.

(2) $\operatorname{ran} B$ is not closed.

(3) $\operatorname{ker} B \neq 0$ and $\operatorname{ran} B=\overline{\operatorname{ran} B} \subset H$.

Corollary 1.2. $B \in L(H)$ is a $C I$ operator if and only if $B^{*} B$ and $B B^{*}$ are invertible or noninvertible together, i.e., $\sigma\left(B^{*} B\right)=\sigma\left(B B^{*}\right)$.

The following corollary is a natural complement to the above results and its proof is straightforward.

Corollary 1.3. Let $B \in L(H)$. If $\operatorname{ker} B=0=\operatorname{ker} B^{*}$, then $B$ is a CI operator. Remark 1.4. From the proofs above, we can also see that the $\mathrm{CI}$ operators can be classified into two parts: (1) there is an $A$ in $L(H)$ such that $A B$ and $B A$ are invertible together (this is the case if and only if $B$ is invertible); and (2) for all $A$ in $L(H), A B$ and $B A$ are always noninvertible (if and only if either $\operatorname{ran} B$ is nonclosed or $\operatorname{ker} B \neq 0$ and $\operatorname{ran} B=\overline{\operatorname{ran} B} \subset H$ ).

Remark 1.5. $B$ is a $\mathrm{CI}$ operator if and only if so is $B^{*}$.

\section{EXAMPLES}

By the preceding results, normal, compact, and invertible operators are immediately examples of $\mathrm{CI}$ operators and so are their products. Next we shall consider their generalizations. 
An operator $B \in L(H)$ such that $\|B x\| \geq\left\|B^{*} x\right\|$ for each $x$ in $H$ is called hyponormal. Obviously, $\operatorname{ker} B \subseteq \operatorname{ker} B^{*}$.

2.1. If $B \in L(H)$ is hyponormal and $\operatorname{ran} B$ is closed, then $B$ is a CI operator if and only if (1) $\operatorname{ker} B \neq 0$, or (2) $\operatorname{ker} B^{*}=0$.

Note that if $\operatorname{ran} B$ is not closed, then, from Theorem $1.1, B$ is a CI operator. Proof. The conclusion can be obtained, when $\operatorname{ker} B \neq 0$, from $\operatorname{ker} B^{*} \supseteq$ $\operatorname{ker} B \neq 0$ and Theorem 1.1 (3), and, when $\operatorname{ker} B^{*}=0$, from $\operatorname{ker} B \subseteq \operatorname{ker} B^{*}=$ 0 and Corollary 1.3 .

If $B$ is a CI operator, then one of the two cases (1) and (3) in Theorem 1.1 must occur. Case (1) implies $\operatorname{ker} B^{*}=0$ and case (3) implies $\operatorname{ker} B \neq 0$.

2.2. If $B \in L(H)$ is hyponormal, then $B$ is a $C I$ operator if and only if either:

(1) $B B^{*}$ is invertible, or

(2) $B^{*} B$ is noninvertible.

Proof. Necessity is trivial.

If $B B^{*}$ is invertible, then it follows from $\operatorname{ran} B \supseteq \operatorname{ran} B B^{*}=H$ and $\operatorname{ker} B \subseteq$ $\operatorname{ker} B^{*}=\operatorname{ker} B B^{*}=0$ that $B$ is invertible, hence $B^{*} B$ is invertible. This also leads to that, if $B^{*} B$ is noninvertible, then so is $B B^{*}$.

Remark 2.3. We say that $B \in L(H)$ is $M$-hyponormal if there is an $M>0$ such that

$$
\left\|(B-\lambda)^{*} x\right\| \leq M\|(B-\lambda) x\|
$$

for all $x \in H$ and all complex number $\lambda$. Clearly, 2.1 and 2.2 remain true for $M$-hyponormal operator $B$ with the proof unchanged.

Since $B \in L(H)$ is an isometry if and only if $B^{*} B=I$, we have

2.4. If $B \in L(H)$ is an isometry, then $B$ is a $C I$ operator if and only if $B$ is unitary.

2.5. If $B$ is invertible, then $B+K$ is a $C I$ operator for every $K \in K(H)$. This follows from ind $(B+K)=$ ind $B=0$. It should be noted that there exist Fredholm operators which are not $C I$ operators.

2.6. If $B \in L(H)$ such that $\sigma(B)$ is a singleton, then $B+K$ is a CI operator for every $K$ in $K(H)$. In particular, if $B$ is a Riesz operator, then $B$ is a $C I$ operator.

Recall that $B$ is a Riesz operator if and only if $\sigma_{e}(B)=0$.

Proof. If $\sigma(B) \neq 0$, then this follows from 2.5. Now suppose that $\sigma(B)=0$. Then $\sigma_{e}(B+K)=0=\sigma_{e}(B)$, which implies that $B+K$ is not semi-Fredholm. By Theorem 2.5 in $[4$, p. 356], we have that either $\operatorname{ran}(B+K)$ is not closed or $\operatorname{dim} \operatorname{ker}(B+K)=\operatorname{dim} \operatorname{ker}(B+K)^{*}=\infty$. Thus, by Theorem $1.1, B+K$ is a CI operator. If $B$ is a Riesz operator, it is known from [5, Theorem 3.3] that $B=C+K$, where $\sigma(C)=0$ and $K \in L(H)$; whence, $B$ is a CI operator.

\section{COMPACT PERTURBATIONS AND NORMALITY}

In this section, we shall give a spectral characterization of the operators which are the products of finitely many normal operators (resp. essentially normal operators). In what follows, $H$ will be a fixed separable complex Hilbert space.

The following Theorem 3.1 is due to [8, Theorem 1.1]. 
Theorem 3.1. If $B \in L(H)$, then the following statements are equivalent:

(1) $B$ is the product of finitely many normal operators.

(2) $\operatorname{dim} \operatorname{ker} B=\operatorname{dim} \operatorname{ker} B^{*}$ or $\operatorname{ran} B$ is not closed.

(3) $B$ is the norm limit of a sequence of invertible operators.

We shall prove

Theorem 3.2. If $B \in L(H)$, then the following conditions are equivalent to those in Theorem 3.1:

(4) $\sigma(A(B+K))=\sigma((B+K) A)$ for every $A$ in $L(H)$ and $K$ in $K(H)$, i.e., $B+K$ is a $C I$ operator for every $K$ in $K(H)$.

(5) $\sigma(A(B+F))=\sigma((B+F) A)$ for every $A$ in $L(H)$ and finite rank operator $F$.

Proof. (5) $\Rightarrow(2)$. If $\operatorname{ran} B$ is closed, we claim that $\operatorname{dim} \operatorname{ker} B=\operatorname{dim} \operatorname{ker} B^{*}$. Otherwise, we may assume that $\operatorname{dim} \operatorname{ker} B<\operatorname{dim} \operatorname{ker} B^{*}$. Then, by Proposition 3.21 in $[4$, p. 366], there exists a finite rank operator $F$ such that $\operatorname{ker}(B+F)=0$ and $\operatorname{ind}(B+F)=\operatorname{ind} B \neq 0$. Therefore, by Theorem $1.1, B+F$ is not a $\mathrm{CI}$ operator, which leads to a contradiction.

(4) $\Rightarrow(5)$ is obvious.

(2) $\Rightarrow(4)$. Let $K \in K(H)$. If $\operatorname{ran}(B+K)$ is not closed, then, by Theorem 1.1, $B+K$ is a $C I$ operator. Now suppose that $\operatorname{ran}(B+K)$ is closed. If $\operatorname{dim} \operatorname{ker}(B+K)<\infty$, then $\operatorname{dim} \operatorname{ker} B<\infty$ and $\operatorname{ran} B$ is closed, thus $\operatorname{dim} \operatorname{ker} B=\operatorname{dim} \operatorname{ker} B^{*}$ which implies $\operatorname{dim} \operatorname{ker}(B+K)=\operatorname{dim} \operatorname{ker}(B+K)^{*}$. If $\operatorname{dim} \operatorname{ker}(B+K)=\infty$, a similar argument shows that $\operatorname{dim} \operatorname{ker}(B+K)^{*}=\infty$. Therefore, in these two cases, $B+K$ is a $\mathrm{CI}$ operator.

Corollary 3.3. If $\sigma(B)$ is a singleton, then $B$ satisfies the conditions in Theorem 3.1.

Corollary 3.4. Let $B \in L(H)$ be an essentially normal operator, i.e. $B^{*} B-B B^{*} \in$ $K(H)$. Then the following statements are equivalent:

(1) There is a normal operator $N$ and a compact operator $K$ such that $B=N+K$.

(2) $p(B)+K$ is a $C I$ operator for any polynomial $p$ and any $K \in K(H)$.

(3) $p(B)$ is the product of finitely many normal operators for any polynomial $p$.

This corollary may be regarded as a new version of the BDF theorem [3].

Proof. (1) $\Rightarrow(2) \Leftrightarrow$ (3) follows from Theorem 3.2 immediately. Now we show that (2) implies (1). By the Brown-Douglas-Fillmore theorem [3], it is sufficient to show that ind $(B-\lambda I)=0$ whenever $\lambda \notin \sigma_{e}(B)$. If there exists $\lambda \notin \sigma_{e}(B)$ such that $\operatorname{ind}(B-\lambda I) \neq 0$, we may assume that $\operatorname{dim} \operatorname{ker}(B-\lambda I)<$ $\operatorname{dim} \operatorname{ker}(B-\lambda I)^{*}$. Then, by Proposition 3.21 in [4, p. 366], there is $K \in K(H)$ such that $\operatorname{ker}(B-\lambda I+K)=0$ and $\operatorname{ind}(B-\lambda I+K)=\operatorname{ind}(B-\lambda I) \neq 0$. Thus, by Theorem 1.1, $B-\lambda I+K$ is not a $\mathrm{Cl}$ operator, which contradicts the assumption.

Remark 3.5. There exists a nonessentially normal operator which satisfies the conditions (2) and (3) of Corollary 3.4. For example, let $B \in L(H)$ such that $\sigma(B)=0$ and $B \notin K(H)$. By $\sigma_{e}(B)=0$ and $B \in K(H), B$ is not essentially normal; but, by Corollary 3.3, $p(B)$ is the product of finitely many normal operators for every polynomial $p$. 
Lemma 3.6. If $\sigma_{e}(A B)=\sigma_{e}(B A)$ for every $A$ in $L(H)$, then one of the following statements holds:

(a) $B$ is the product of finitely many normal operators.

(b) $B$ is a Fredholm operator.

Proof. From Theorem 3.1, it is sufficient to show that if $B$ is semi-Fredholm, then $B$ is Fredholm.

We may assume that $\operatorname{dim} \operatorname{ker} B<\infty$ and $\operatorname{ran} B$ is closed. Then there is $A \in L(H)$ such that $A B-I \in K(H)$; thus, $0 \notin \sigma_{e}(A B)=\sigma_{e}(B A)$, which implies that $B$ is Fredholm.

The following theorem, an analogue of Theorem 3.2 in the Calkin algebra, gives a spectral characterization of compact perturbations of the product of finitely many essentially normal operators.

Theorem 3.7. Let $B \in L(H)$. Then the following statements are equivalent:

(1) $\sigma_{e}(A B)=\sigma_{e}(B A)$ for every $A$ in $L(H)$.

(2) $B$ is a compact perturbation of the product of finitely many essentially normal operators; i.e., $\pi(B)$ is the product of finitely many normal elements in $L(H) / K(H)$.

(3) $B$ is the norm limit of a sequence of Fredholm operators; i.e., $\pi(B)$ is the norm limit of invertible elements in $L(H) / K(H)$, where $\pi$ is the canonical map of $L(H)$ onto $L(H) / K(H)$.

Proof. From [1, Theorem 4] we have that (3) is equivalent to the condition that $\operatorname{dim} \operatorname{ker} B=\operatorname{dim} \operatorname{ker} B^{*}, \operatorname{ran} B$ is not closed, or $B$ is Fredholm. Thus (1) implies (3) by Lemma 3.6.

$(3) \Rightarrow(2)$. It is sufficient to prove that, if $B$ is Fredholm, then $B$ must satisfy (2).

Let $\phi$ be a faithful unital *-representation of the Calkin algebra $L(H) / K(H)$ on a Hilbert space $H^{\prime}$. Then $\phi(\pi(B))$ is invertible in $\operatorname{ran} \phi$. Let $R=$ $\left(\phi(\pi(B))^{*} \phi(\pi(B))\right)^{1 / 2} \in \operatorname{ran} \phi$ and $S=\phi(\pi(B)) R^{-1} \in \operatorname{ran} \phi$. Then $S^{*} S=$ $S S^{*}=I$, and thus $\phi(\pi(B))=S R$ is the product of normal operators. If $\pi\left(B_{1}\right)=\phi^{-1}(S), \pi\left(B_{2}\right)=\phi^{-1}(R)$, then $B_{1}$ and $B_{2}$ are essentially normal operators and $\pi(B)=\pi\left(B_{1} B_{2}\right)$. Therefore, $B$ satisfies (2),

$(2) \Rightarrow(1)$. Obviously, we may suppose that $B$ is essentially normal. Let $\phi$ be as above. Then $\phi(\pi(B))$ is normal in $L\left(H^{\prime}\right)$, and thus $\sigma(\phi(\pi(B)) C)=$ $\sigma(C \phi(\pi(B)))$ for every $C \in L\left(H^{\prime}\right)$. In particular, $\sigma_{e}(A B)=\sigma(\pi(A B))=$ $\sigma(\phi(\pi(A B)))=\sigma(\phi(\pi(A)) \phi(\pi(B)))=\sigma(\phi(\pi(B)) \phi(\pi(A)))=\sigma(\pi(B A))=$ $\sigma_{e}(B A)$ for every $A$ in $L(H)$. Thus (1) holds.

\section{ACKNOWLEDGMENT}

The authors are grateful to the referee for providing several suggestions.

\section{REFERENCES}

1. R. Bouldin, The essential minimum modulus, Indiana Univ. Math. J. 30 (1981), 514-517.

2. _ The product of operators with closed range, Tôhoku Math. J. 25 (1973), 359-363.

3. L. Brown, R. Douglas, and P. Fillmore, Unitary equivalence modulo the compact operators and extensions of $C^{*}$-algebras, Proc. Conf. Operator Theory, Lecture Notes in Math., vol. 345, Springer-Verlag, New York, 1973, pp. 58-128. 
4. J. B. Conway, A course in functional analysis, Springer-Verlag, New York, 1985.

5. H. R. Dowson, Spectral theory of linear operators, London Math. Soc. Monographs (N.S.), vol. 12, Academic Press, New York, 1978.

6. M. Hladnik and M. Omladič, Spectrum of the product of operators, Proc. Amer. Math. Soc. 102 (1988), 300-302.

7. A. E. Taylor and D. C. Lay, Introduction to functional analysis, Wiley, New York, 1980.

8. P. Y. Wu, Products of normal operators, Canad. J. Math. 40 (1988), 1322-1330.

Department of Mathematics, Qufu Normal University, Qufu, 273165, Shandong, PeoPLE'S REPUBLIC OF CHINA 